\title{
OPTICAL FLOW BY MULTI-SCALE ANNOTATED KEYPOINTS A Biological Approach
}

\author{
Miguel Farrajota, J.M.F. Rodrigues and J.M.H. du Buf \\ Vision Laboratory, Institute for Systems and Robotics (ISR), University of the Algarve (ISE and FCT), 8005-139 Faro, Portugal \\ elsio_farrajota@hotmail.com, \{jrodrig,dubuf $\} @$ ualg.pt
}

Keywords: Optical flow, multi-scale, keypoint classification, visual cortex, segregation, tracking.

\begin{abstract}
Optical flow is the pattern of apparent motion of objects in a visual scene and the relative motion, or egomotion, of the observer in the scene. In this paper we present a new cortical model for optical flow. This model is based on simple, complex and end-stopped cells. Responses of end-stopped cells serve to detect keypoints and those of simple cells are used to detect orientations of underlying structures and to classify the junction type. By combining a hierarchical, multi-scale tree structure and saliency maps, moving objects can be segregated, their movement can be obtained, and they can be tracked over time. We also show that optical flow at coarse scales suffices to determine egomotion. The model is discussed in the context of an integrated cortical architecture which includes disparity in stereo vision.
\end{abstract}

\section{INTRODUCTION}

Optical flow, also called optic flow, is the motion pattern caused by moving objects in a visual scene. It can be described by motion or displacement vectors of entire objects or parts of them between successive time frames. In the case of egomotion, i.e., the eye of a moving person or a moving camera, the relative motion between observer and scene also contributes.

Experiments have strengthened the arguments that neurons in a specialised region of the cerebral cortex play a major role in flow analysis (Wurtz, 1998), that neuronal responses to flow are shaped by visual strategies for steering (William and Charles, 2008), and that flow processing has an important role in the detection and estimation of scene-relative object movements during egomotion (Warren and Rushton, 2009). For the latter, the brain identifies and globally discounts (i.e., subtracts) optical flow patterns across the visual scene, a process called flow parsing.

Morrone et al. (2000) demonstrated that neurons in area V5/MT (medial temporal) respond selectively to components of optical flow, such as circular and radial motion. Smith et al. (2006) showed that neurons in area MST (middle superior temporal) seem to be more selective to complex movements than those in area MT, the latter being more devoted to simple movements, although both areas respond to all motion stimuli but with different activation patterns. Although many cells may respond to more than one type of motion stimulus, individual cells show different direction selectivities (Duffy and Wurtz, 1991). In addition, cells in area MST were reported to be selective for rotation and expansion (Orban et al., 1992), having larger receptive fields and less precise retinotopic mapping than those in area MT. Therefore, MST cells convey more global information about a scene's structure and motions (Smith et al., 2006).

An essential function of visual processing is to establish the position of the body in space and, in concert with the other sensory systems, to monitor its movements: egomotion through optical flow (Wall and Smith, 2008). For example, forward motion generates an expanding flow pattern on the retinae and, with eyes fixated centrally, the heading direction corresponds to the centre of expansion. Area MST being sensitive to more global optical flow patterns, it has been suggested that MST has a central role in guiding heading in macaques. The same authors identified two areas of the human brain which represent visual cues to egomotion more directly than does area MST. One is the putative area VIP in the anterior part 
of the intraparietal sulcus. The other is a new visual area coined cingulate sulcus visual area $(\mathrm{CSv})$. In contrast to these new areas, areas V1 to V4 and MT respond about equally to stimuli mimicking arbitrary motion and egomotion, whereas area MST has intermediate properties, responding well to various motion stimuli but with a modest preference for egomotioncompatible stimuli.

Apart from motion processing, we know that the visual cortex detects and recognises objects by means of the ventral "what" and dorsal "where" subsystems. Both bottom-up (visual input code) and top-down (expected object and position) data streams are necessary for obtaining size, rotation and translation invariance, assuming that object templates are normalised in visual memory.

Recently we presented cortical models based on multi-scale line/edge and keypoint representations (Rodrigues and du Buf, 2006, 2009b). These representations, all based on responses of simple, complex and end-stopped cells in V1, can be integrated for different processes: visual reconstruction or brightness perception, focus-of-attention (FoA), object segregation and categorisation, and object and face recognition. The integration of FoA, region segregation and object categorisation is important for developing fast gist vision, i.e., which types of objects are about where in a scene.

Optical flow, as for disparity in stereo vision, complements colour and texture in object segregation, possibly in, but not necessarily limited to, the dorsal "where" pathway where keypoints may play a major role in FoA (Rodrigues and du Buf, 2006). In this paper we present a new model for cortical optical flow which is based on annotated (classified) multi-scale keypoints. We show that the information can be used for egomotion and for object segregation and tracking.

In Section 2 we present multi-scale keypoint detection and annotation, in Section 3 optical flow detection, in Section 4 object tracking using optical flow information, and we conclude with a final discussion and lines for future work in Section 5.

\section{MULTI-SCALE KEYPOINT ANNOTATION}

Keypoints are based on end-stopped cells (Rodrigues and du Buf, 2006). They provide important information because they code local image complexity. Moreover, since keypoints are caused by line and edge juntions, detected keypoints can be classified by the underlying vertex structure, such as K, L, T, + etc. This is very useful for most if not all matching problems: object recognition, stereo disparity and optical flow. In this section we describe the multi-scale keypoint detection and annotation processes.

\subsection{KEYPOINT DETECTION}

Gabor quadrature filters provide a model of cortical simple cells (Rodrigues and du Buf, 2006). In the spatial domain $(x, y)$ they consist of a real cosine and an imaginary sine, both with a Gaussian envelope. Responses of even and odd simple cells, which correspond to real and imaginary parts of a Gabor filter, are obtained by convolving the input image with the filter kernel, and are denoted by $R_{s, i}^{E}(x, y)$ and $R_{s, i}^{O}(x, y), s$ being the scale, $i$ the orientation $\left(\theta_{i}=\right.$ $\left.i \pi / N_{\theta}\right)$ and $N_{\theta}$ the number of orientations (here 8) with $i=\left[0, N_{\theta}-1\right]$. Responses of complex cells are then modelled by the modulus

$$
C_{s, i}(x, y)=\left[\left\{R_{s, i}^{E}(x, y)\right\}^{2}+\left\{R_{s, i}^{O}(x, y)\right\}^{2}\right]^{1 / 2} .
$$

There are two types of end-stopped cells, single and double. These are applied to $C_{s, i}$ and are combined with tangential and radial inhibition schemes in order to obtain precise keypoint maps $K_{s}(x, y)$. For a detailed explanation with illustrations see Rodrigues and du Buf (2006). Below, the scale of analysis will be given by $\lambda$ expressed in pixels, where $\lambda=1$ corresponds to 1 pixel.

Figure 1 (top-left) shows a scene with, on the second row from top, keypoints detected (diamond symbols) at two scales $\lambda=6$ (left) and 27 (right). At top-right it shows one quadrant of a test image with a black square against a homogeneous background (top-left) and a noisy background (top-right), both with a correctly detected keypoint at the junction. All other images show annotated keypoints; see below.

\subsection{KEYPOINT ANNOTATION}

In order to classify any detected keypoint, the responses of simple cells $R_{s, i}^{E}$ and $R_{s, i}^{O}$ are analysed, but now using $N_{\phi}=2 N_{\theta}$ orientations, $\phi_{k}=k \pi / N_{\theta}$ and $k=\left[0, N_{\phi}-1\right]$. This means that for each simplecell orientation on $[0, \pi]$ there are two opposite analysis orientations on $[0,2 \pi]$, e.g., $\theta_{1}=\pi / N_{\theta}$ results in $\phi_{1}=\pi / N_{\theta}$ and $\phi_{9}=9 \pi / N_{\theta}$; see Fig. 2 (top).

This division into response-analysis orientations is acceptable, according to Hubel (1995), because a typical cell has a maximum response at some orientation and its response decreases on both sides, from 10 to 20 degrees, after which it declines steeply to zero; see also du Buf (1993). In addition, this division is a 


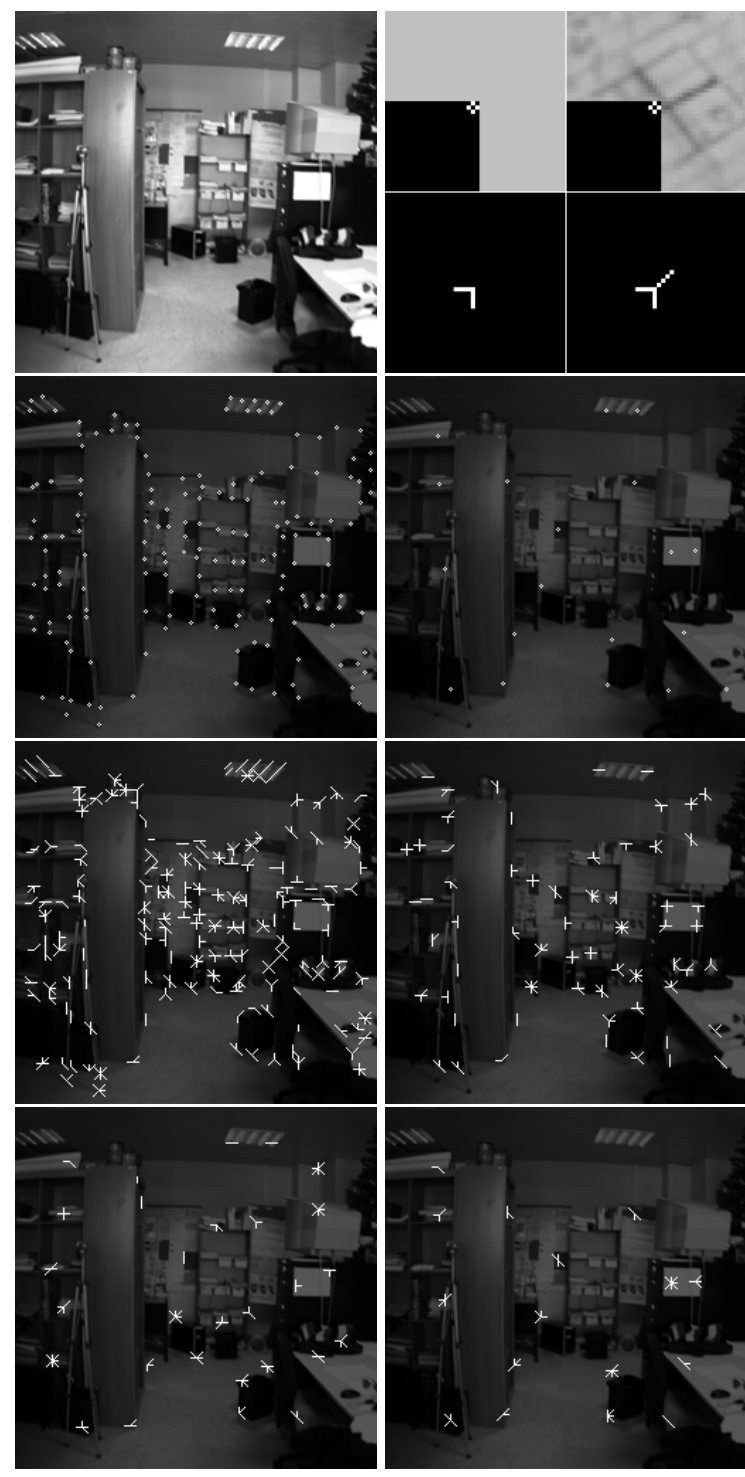

Figure 1: Keypoint detection and annotation. Input scene (top-left) with, on the 2nd row, keypoints detected at scales $\lambda=6$ (left) and 27 (right). The 3rd and 4th rows show annotated keypoints at scales $\lambda=\{6,12,18,27\}$. The top-right image shows one quadrant of a black square against a homogeneous background (left) and noisy background (right), both at $\lambda=6$.

compromise between the cost (CPU time) of the number of orientations and the accuracy of the results.

Classifying keypoints is not trivial, because responses of simple and complex cells, which code the underlying lines and edges at the vertices, are unreliable due to response interference effects (du Buf, 1993). This implies that responses must be analysed in a neighbourhood around each keypoint, and the size of the neighbourhood must be proportional to the scale of the cells. The validation of the line and edge orientations which contribute to the vertex structure is based on an analysis of the responses of simple cells, both $R_{s, j}^{E}$ and $R_{s, j}^{O}$, and consists of three steps: (1) only responses with small variations at three distances are considered, (2) local maxima of the responses over orientations are probed and the remaining orientations are inhibited, and (3) responses of even and odd simple cells are matched in order to keep the orientations which are common to both.

In step (1), at any scale and each orientation $\phi_{k}$ the responses of the simple cells on three circles around the keypoint position, with radii $\lambda / 2, \lambda$ and $2 \lambda$, are compared. Instead of only taking the responses at $\phi_{k}$, the orientation intervals $\phi_{k} \pm \pi / N_{\phi}$ are considered. The three maximum responses of $R_{k, r}^{E / O}$ in the orientation interval around $k$ and at the three radii $r$ are detected, and their maximum $\hat{R}_{k}=\max _{r} R_{k, r}^{E / O}$. Only responses with small variations at the three radii are considered $\left(R>0.6 \hat{R}_{k}\right)$, yielding $N_{\beta}$ candidate orientations. The smallest radius of $\lambda / 2$ was chosen because of the interference effects referred to above (du Buf, 1993). The other two radii were determined experimentally.

Biologically, the above process is based on clusters of grouping cells with dendritic fields (Fig. 2 (top) in red) covering the orientation intervals at each of the three radii. These grouping cells combine other cells with self-inhibition for non-maximum suppression. The three grouping cells at the three radii feed into another grouping cell which compares the responses and which inhibits itself when the responses are not similar. Figure 2 (bottom) shows responses of simple cells in the case of a black square against a noisy background (Fig. 1 top-right). It shows two scales, $\lambda=6$ (column 1 and 2 ) and $\lambda=15$ (column 3 and 4), only three of all eight orientations (top to bottom), even cells in columns 1 and 3 and odd cells in columns 2 and 4. Dark levels are negative and bright ones are positive. Also shown is one detected keypoint at each scale with, in red, the three circles at $\lambda / 2, \lambda$ and $2 \lambda$ at which the grouping cells are located. The drawing at the top shows the orientation intervals, also in red, covered by the dendritic fields in the case of $\theta_{1}$ with opposite orientations $\phi_{1}$ and $\phi_{9}$.

In step (2), the responses at the detected orientations are summed, $\bar{R}=\Sigma_{k} R_{k}$, and, for validation purposes, all responses $\hat{R}_{k}$ below a threshold value of $\bar{R}$ are suppressed $\left(0.95 \bar{R} / N_{\beta}\right)$. Biologically, this is done by another grouping cell which sums responses of the grouping cells in step (1) and which may inhibit the same cells if their response is too low.

If there also exist maximum responses $\hat{R}_{k}$ at the two neighbouring orientations $\phi_{k-1}$ and $\phi_{k+1}$, they will be inhibited if they are too low $\left(\hat{R}_{k \pm 1}<0.95 \hat{R}_{k}\right)$. The above values were determined by analysing many 

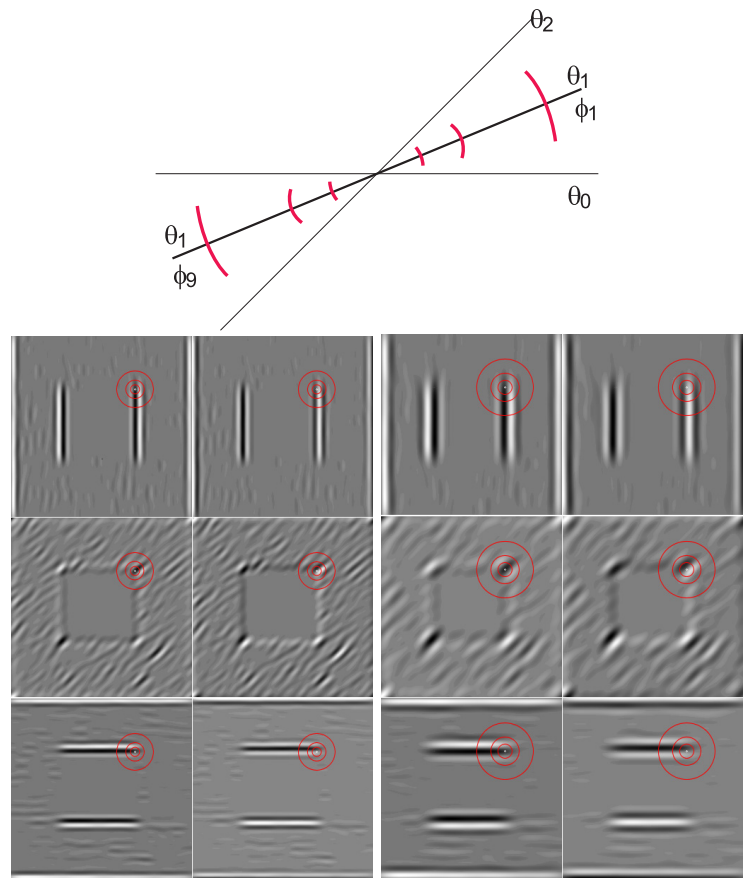

Figure 2: Top: a few orientations of simple cells $(\theta)$ and opposing orientations for keypoint classification $(\phi)$ plus, in red, orientation intervals covered by grouping cells. Bottom: responses of simple cells at 3 orientations (top to bottom) and at two scales $\lambda=6$ (left) and $\lambda=15$ (right). From left to right: responses of even and odd simple cells. Also shown is one detected keypoint with, in red, the 3 circles on which the responses are analysed for keypoint annotation.

objects like triangles, squares and polygons.

Step (2) is necessary because we need the orientations which convey the most consistent information, i.e., not being due to varying lighting levels, light sources casting shadows, background structures and even dynamic backgrounds like the wind playing the crowns of trees. Figure 1 (top-right) shows the difference in the case of the same black square against a homogeneous background (left) and a structured one (right). The diagonal structure in the background has a much lower contrast than the edge of the square. Hence, without step (2) the keypoint would have been annotated by three orientations instead of two.

The analysis in step (3) only concerns the matching of equal orientations, i.e., inhibiting all orientations which have not been detected in the responses of both even $\left(R_{s, j}^{E}\right)$ and odd $\left(R_{s, j}^{O}\right)$ simple cells. Remaining orientations $\phi_{k}$ are attributed to the keypoint, plus the junction type K, L, T, +, etc. Again, the matching is achieved by grouping cells which combine the grouping cells devoted to $R_{s, j}^{E}$ and $R_{s, j}^{O}$.

In the above procedure there is only one exception: keypoints at isolated points and blobs, especially at very coarse scales, are also detected but they are not caused by any line/edge junctions. Such keypoints are labeled "blob" without attributed orientations.

The bottom four images in Fig. 1 show results of keypoint annotation at the four scales $\lambda=$ $\{6,12,18,27\}$. At fine scale there are many keypoints and at coarse scale less. Below, the annotated keypoints will be exploited in different processes. As mentioned above, keypoint detection may occur in cortical areas V1 and V2, whereas keypoint annotation requires bigger receptive fields and could occur in V4. Optical flow is then processed in areas V5/MT and MST.

\section{OPTICAL FLOW}

Optical flow is determined by matching annotated keypoints in successive camera frames, but only by matching keypoints which may belong to the same object. To this purpose we use regions defined by saliency maps. Moreover, we do not consider all scales independently, for two reasons: (1) nonrelevant areas of an image can be skipped because of the hierarchical scale structure, and (2) by applying a hierarchical tree structure, the accuracy of the matching can be increased, therefore also increasing the accuracy of the optical flow. The latter idea is based on the strategies as employed in our visual system (Rodrigues and du Buf, 2009a; Bar, 2004).

\subsection{OBJECT SEGREGATION}

We apply a multi-scale tree structure in which at a very coarse scale a root keypoint defines a single object, and at progressively finer scales more keypoints are added which convey the object's details. However, coarser scales imply bigger filter kernels and more CPU time, so for practical reasons the coarsest scale applied here will be $\lambda=27$, which is a compromise between speed and quality of results.

Below we use $\lambda=[6,27]$ with $\Delta \lambda=3$, and at the moment all keypoints at $\lambda=27$ are supposed to represent individual objects, although we know that it is possible that several of those keypoints may belong to a same object. Each keypoint at a coarse scale is related to one or more keypoints at one finer scale, which can be slightly displaced. This relation is modelled by down-projection using grouping cells with a circular axonic field, the size of which $(\lambda)$ defines the region of influence. A responding keypoint cell activates a grouping cell. Only if the grouping cell is also excitated by responding keypoint cells at one level lower (the next finer scale), a grouping cell at the lower level is activated. This is repeated until the 


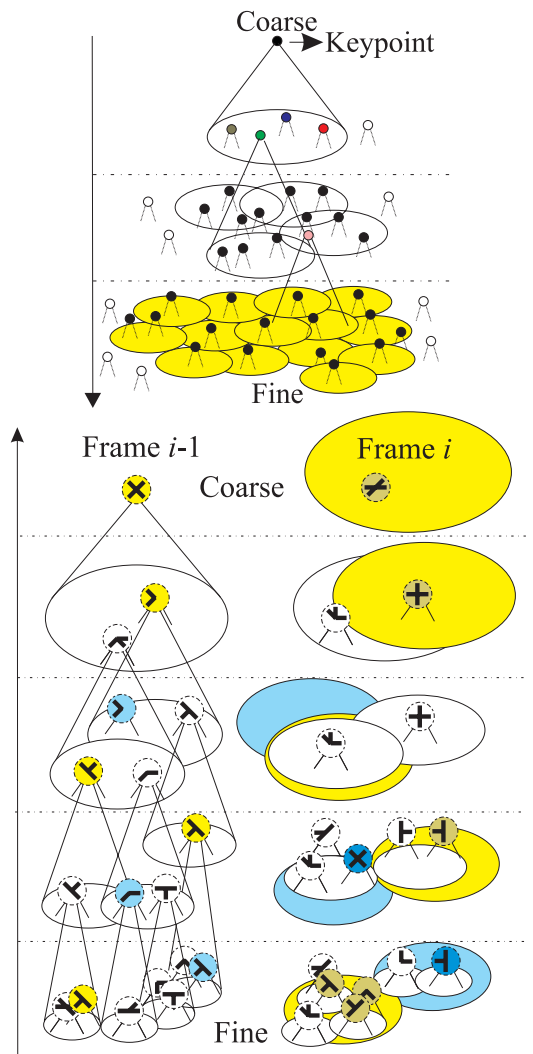

Figure 3: Top: hierarchical tree structure over scales. Bottom: matching of annotated keypoints between successive frames; see text for details.

finest scale has been reached. By doing so, all keypoints outside the areas of influence of the grouping cells will not be considered, thus avoiding unnecessary computations. Figure 3 (top) illustrates the principle of the linking process with cones representing the axonic fields of the grouping cells. At the finest scale the region of influence of the keypoint at the coarsest scale is indicated by the yellow area.

As mentioned above, at a very coarse scale each keypoint - or central keypoint CKP - should correspond to an individual object. However, at the coarsest scale applied, $\lambda=27$, this may not be the case and an object may cause several keypoints. In order to determine which keypoints could belong to the same object we combine saliency maps with the multi-scale tree structure.

A saliency map can be based on keypoints as these code local image complexity (Rodrigues and du Buf, 2006). Such a map is created by summing detected keypoints over all scales $s$, such that keypoints which are stable over scale intervals yield high peaks, but in order to connect the individual peaks and yield regions a relaxation area is applied. As applied above, the area is proportional to the scale and has a ra- dius of $\lambda$. Here, we simplify the computation of saliency maps by summing responses of end-stopped cells at all scales, which yields similar results. Figure 6 (right) shows on the 2nd to the 4th row examples of such saliency maps which correspond to the input frames to their left. For illustration purposes the maps were scaled to the interval $[0,255]$. The maps will be thresholded in order to obtain segregated regions; see below.

\subsection{KEYPOINT MATCHING}

At this point we have, for each frame, the tree structure which links the keypoints over scales, from coarse to fine, with associated regions of influence at the finest scale. We also have the saliency map by summing responses of end-stopped cells over all scales. The latter, after thresholding, yields segregated regions which are intersected with the regions of influence of the tree. Therefore, the intersected regions link keypoints at the finest scale to segregated regions which are supposed to represent individual objects.

Now, each annotated keypoint of frame $i$ can be compared with all annotated keypoints in frame $i-1$. This is done at all scales, but the comparison is restricted to an area with radius $2 \lambda$ instead of $\lambda$ at each scale in order to allow for larger translations and rotations. In addition: (1) at fine scales many keypoints outside the area can be skipped since they are not likely to match over large distances, and (2) at coarse scales there are less keypoints, $\lambda$ is bigger and therefore larger distances (motions) are represented there. The tree structure is built top-down, Fig. 3 (top), but the matching process, Fig. 3 (bottom), is bottom-up: it starts at the finest scale because there the accuracy of the keypoint annotation is better. Keypoints are matched by combining three similarity criteria with different weight factors: the distance $D$, the attributed orientations $O$, and the tree correspondence $C$.

The distance $D$ serves to emphasise keypoints which are closer to the centre of the matching area. For having $D=1$ at the centre and $D=0$ at radius $2 \lambda$, we use $D=(2 \lambda-d) / 2 \lambda$ with $d$ the Euclidean distance. Biologically, there may be no need to use Euclidean distances if a kind of dynamic feature routing in space and time is used, possibly with motion prediction in the "where" pathway. ${ }^{1}$ Dynamic routing from frame $i-1$ to frame $i$, possibly also involving previous frames $i-2$ etc., is a spatiotemporal map-

\footnotetext{
${ }^{1}$ Motion prediction is a form of adaptation which could explain the motion aftereffect, for example our illusion that a railway station moves after our train has stopped. This may occur in area MT (Kohn and Movshon, 2003).
} 


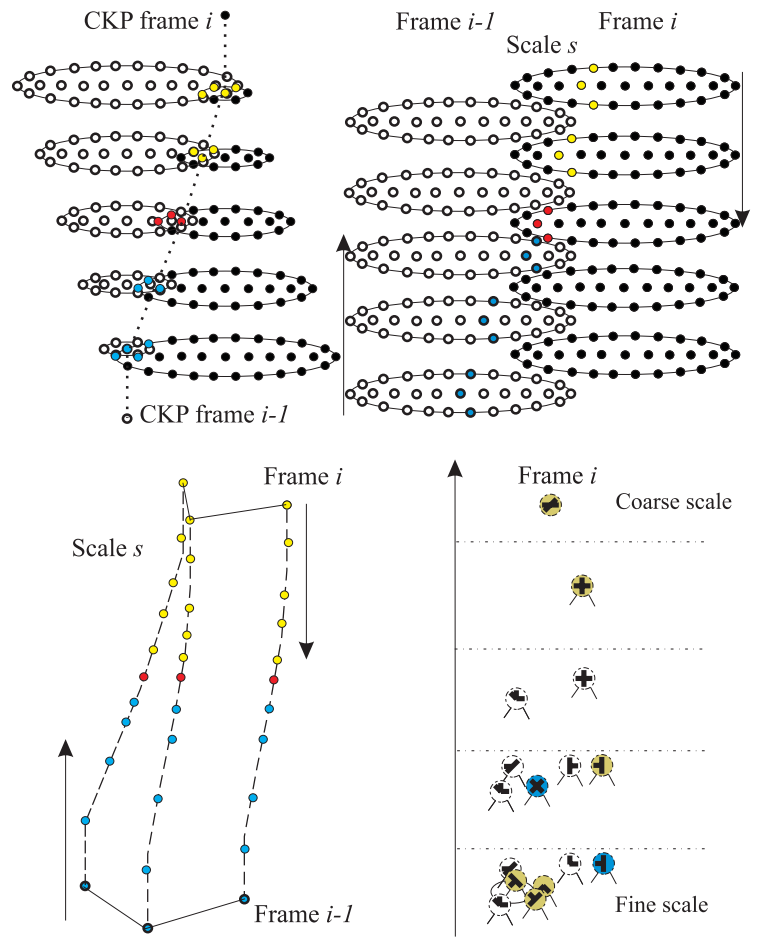

Figure 4: Top: dynamic routing between coarse keypoints of successive frames (left), and cell representation of distance D (right). Bottom: cell representation of orientation $\mathrm{O}$ (left), and tree correspondence C (right); see text for details.

ping, assuming a stack of neural layers in which a few previous maps are stored: a new frame is always pushed on the "top-of-stack" and older frames are also being pushed down. As for dynamic routing in invariant object recognition, see Rodrigues and du Buf (2009a), the principle can be based on linking first keypoints at very coarse scales (central keypoints or CKP in Fig. 4 top-left) in space after which finer scales refine the linking (Fig. 4 top-right). This is subject to ongoing research.

The orientation error $O$ measures the differences of the attributed orientations, but with a relaxation of $\pm \pi / N_{\theta}$ of all orientations such that also a small rotation of the vertex structure is allowed. Similar to $D$, the summed differences are combined such that $O=1$ indicates good correspondence and $O=0$ a lack of correspondence. Obviously, keypoints marked "blob" do not have orientations and are treated separately. Biologically, the orientation error could be based on the number of intermediate layers in the routing which is necessary to establish correspondence of the vertex structure, which is shown simplified in Fig. 4 (bottom-left).

Parameter $C$ measures the number of matched keypoints at finer scales, i.e., at any scale coarser than the finest one. The keypoint candidates to be matched in frame $i$ and in the area with radius $2 \lambda$ are linked in the tree to localised sets of keypoints at all finer scales. The number of linked keypoints which have been matched is divided by the total number of linked keypoints. This is achieved by sets of grouping cells at all but the finest scale which sum the number of linked keypoints in the tree, both matched and all. Hence, parameter $C$ describes the consistency of the matching at a candidate's position at the finer scales, thereby influencing the matching of the candidate at the actual scale. Figure 4 (bottom-right) illustrates in colour the matched keypoints which influence the matching at coarser scales; see also Fig. 3 (bottom).

The three parameters are combined by grouping cells which can establish a link between keypoints in frame $i-1$ and $i$. Mathematically we use the similarity measure $S=\alpha O+\beta C+\gamma D$, with $\alpha=0.4, \beta=0.3$ and $\gamma=0.3$. These values were determined empirically. The candidate keypoint with the highest value of $S$ in the area $(2 \lambda)$ is selected and the vector between the keypoint in frame $i-1$ and the matched one in frame $i$ is computed. The remaining candidates in the area can be matched to other keypoints in frame $i-1$, provided they are in their local area. Keypoints which cannot be matched are discarded.

Figure 5 (top-left) shows a sequence of 10 frames with a moving and rotating star combined into one image and, at right, examples of keypoints detected at scales $\lambda=6$ (left) and 24 (right), together with their annotation below. The 2nd row (left) shows the optical flow vectors from the first to the last frame. The other images show two superimposed frames with egomotion of the camera and the optical flow vectors at four scales $\lambda=\{6,12,18,27\}$. It can be seen that there are some but few errors. In principle, such outliers can be removed, but important is that the correct egomotion can be seen at all scales with, as expected, more variation at the finest scale. In fact, the flow detected at the coarsest scale (bottom-right) is already sufficient to extract the egomotion. This is consistent with object categorisation and recognition (Bar, 2004; Oliva and Torralba, 2006; Rodrigues and du Buf, 2009b): coarse-scale information is available first and this is used for a first but rough categorisation $(80-100 \mathrm{~ms})$, after which information at finer scales becomes available to refine the categorisation.

After obtaining egomotion vectors at the coarsest scale, by averaging but perhaps after elimination of outliers in local neighbourhoods because of possible camera rotation, egomotion can be eliminated by subtracting this information from all flow vectors (Warren and Rushton, 2009). Bearing this in mind, when we compute the tree correspondence $C$ for keypoint matching at only the coarsest scale, the result will be 


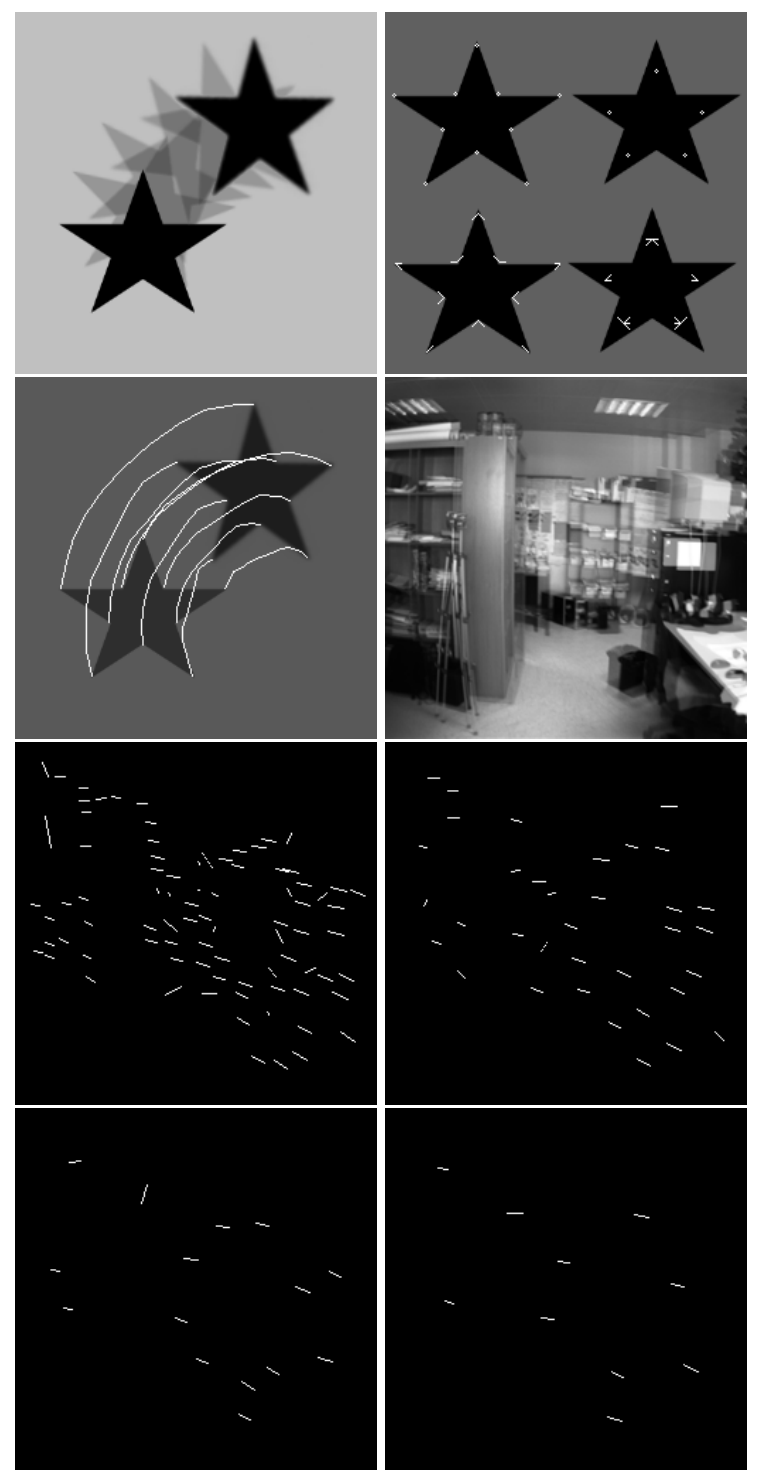

Figure 5: Optical flow results. Top-left: frames with a moving and rotating star combined into one image. Top-right: detected keypoints at scales $\lambda=6$ (left) and 24 (right), with the annotated keypoints below. Second from top, at left, optical flow vectors. The other images show two combined frames with egomotion of the camera and optical flow vectors at scales $\lambda=\{6,12,18,27\}$.

similar. However, the result will be more robust if we use all scales, as in the object categorisation and recognition process (Rodrigues and du Buf, 2009b).

\section{TRACKING OF OBJECTS}

After thresholding, the saliency map of a frame yields separated regions-of-interest (RoI); see Fig. 6, the 2nd to 4 th row at right. These regions can be intersected with the regions as defined by the tree structure, in Fig. 3 (top) shown in yellow. The regions of the dendritic fields of the end-stopped cells which generate the saliency map will overlap the regions of the axonic fields of the keypoint-grouping cells in the tree structure. Hence, neighbouring keypoints are grouped together in the RoIs and their displacement vectors after the matching process yield the optical flow of segregated image regions, i.e., where an individual object or a combination of connected (sub)objects is or are moving. In order to discard very small optical flow due to the camera's motion (not significant egomotion; see above) and to obtain more robust results, optical flow vectors are only computed if at at least four scales the matched keypoints in successive frames have displacement vectors with a length which is bigger than one pixel.

Figure 6 (top) shows a montage of a sequence with a moving robot car (at left) and the tracked optical flow vectors (at right): in white for $\lambda=6$ and in red for $\lambda=27$. Rows 2 to 4 show frames with the robot car segregated in a box (at left) and the corresponding saliency maps (at right). The bottom row shows zooms of two frames with flow vectors at scale $\lambda=9$.

Figure 7 shows two more sequences with moving persons. The top sequence shows a moving person far away, with the bounding box and tracked motion (arrows). The bottom sequence shows a person at close range, in which case the different motions of the different body parts can be distinguished, also the motion of the shadow. The bottom sequence illustrates a real application: detecting and tracking moving obstacles on paths and sidewalks, which is for a navigation aid for the blind in the context of the SmartVision project. In this case the optical flow is complemented by the borders of the path and their intersection in the vanishing point, and the tracking of the centre of the bounding box relative to the vanishing point can be used to detect if the obstacle is approaching or not, for obstacle avoidence.

\section{CONCLUSIONS}

In a previous paper we have shown that keypoint scale-space provides very useful information for constructing saliency maps for Focus-of-Attention (FoA), and that faces can be detected by grouping facial landmarks defined by keypoints at eyes, nose and mouth (Rodrigues and du Buf, 2006). We have also shown that line/edge scale-space provides very useful information for face and object recognition (Rodrigues and du Buf, 2009b). Obviously, object detection and 


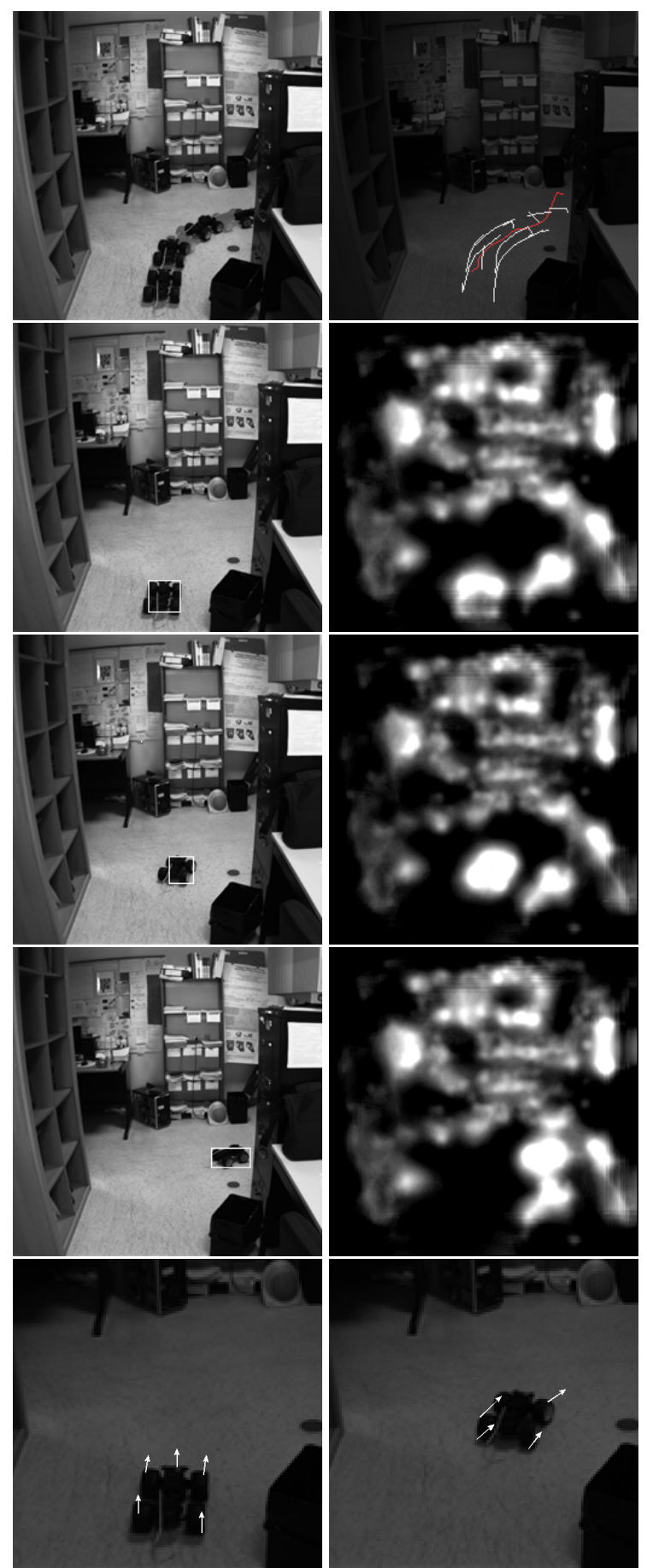

Figure 6: Top: a sequence with a moving robot car (left) and combined optical flow vectors (right), in white at a fine scale and in red at a coarse scale. Rows 2 to 4 show frames with the robot car segregated in a box (left) and the corresponding saliency maps (right). The bottom row shows zooms of optical flow vectors at scale $\lambda=9$.

recognition are related processes, with a seamless integration in the where and what pathways. However,
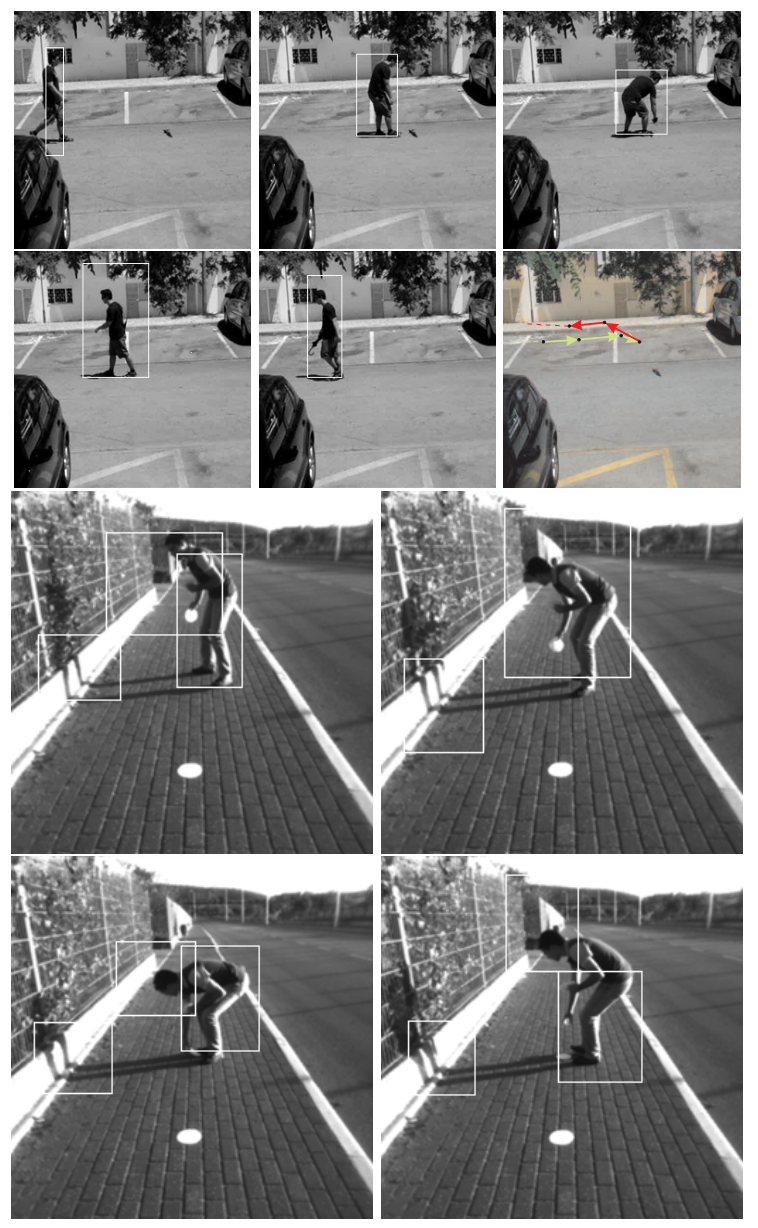

Figure 7: Two sequences with moving persons. The persons have been segregated (bounding box) and tracked (top sequence; arrows), and differently moving body parts have been detected, including shadows (bottom sequence).

there is no (known) dichotomy in the sense that keypoints are only used in the where pathway and lines and edges only in the what pathway.

In this paper we showed that keypoint detection can be complemented by keypoint annotation, and that annotated keypoints in a hierarchical tree structure can be used for keypoint matching in order to obtain optical flow. In addition, since local clusters of keypoints are mostly related to individual moving objects, object segregation can be achieved and objects can be tracked. As written before, cortical areas MT and MST are involved in optical flow and egomotion, but recent results obtained with fMRI showed no clear neural activity in their ventral (what) and dorsal (where) subregions, but elevated activity in between the subregions (Smith et al., 2006). This might indicate that optical flow at MT level is processed separately or involves both pathways. The fact that optical flow can be used to obtain object segrega- 
tion, as demonstrated here, in addition to our previous experiments concerning saliency maps for FoA and face detection, in all cases only using keypoint scale space, would indicate some "preference" of the dorsal (where) pathway for keypoints. This idea is strengthened by the fact that area MT also plays a role in the motion-aftereffect illusion (Kohn and Movshon, 2003), which is tightly related to motion adaptation and prediction. Therefore, motion prediction might play a very important role in the dorsal pathway, not only where objects are now but also where they are expected next. Such predictions tied to objects may lead to much more efficient processing, for example in robot vision, because most image regions can be skipped. Nevertheless, robot vision also requires some sort of "arousal" system for spotting new or unexpected moving objects.

Having a model for matching keypoints in consecutive time frames for optical flow, the same principle can be applied to stereo (disparity), matching left and right frames. Since information of one of the two frames is already available for optical flow, the required additional CPU time will be limited, especially if only the distance of moving objects is necessary, for example to detect objects which may be on collision course, with and without egomotion. In general, however, disparity can be used for obtaining a 3D sketch of an entire scene, plus the 3D structure of individual objects in the scene which may complement the (2D) line/edge scale space for object recognition. Moreover, optical flow and disparity can be combined to obtain more robust object segregations.

Keypoints can complement the line/edge coding in attributing depth, not only to vertical lines and edges but also line and edge junctions. This results in a sort of 3D "wireframe" representation as used in modelling solid objects in computer graphics. The fact that projections from left and right eyes are very close in the cortical hypercolumns and that many simple and complex cells are also disparity tuned suggests that our visual system processes $3 \mathrm{D}$ objects in the same way, probably simplifying 3D object recognition.

\section{ACKNOWLEDGEMENTS}

Portuguese Foundation for Science and Technology (FCT) through the pluri-annual funding of the Inst. for Systems and Robotics (ISR/IST), the POS_Conhecimento Program with FEDER funds, and FCT project SmartVision (PTDC/EIA/73633/2006).

\section{REFERENCES}

Bar, M. (2004). Visual objects in context. Nature Rev.: Neuroscience, 5:619-629.

du Buf, J. (1993). Responses of simple cells: events, interferences, and ambiguities. Biol. Cybern., 68:321-333.

Duffy, C. and Wurtz, R. (1991). Sensitivity of mst neurons to optic flow stimuli. I. A continuum of response selectivity to large-field stimuli. J. Neurophysiol., 65(6):1329-1345.

Hubel, D. (1995). Eye, Brain and Vision. Scientific American Library.

Kohn, A. and Movshon, J. (2003). Neuronal adaptation to visual motion in area $\mathrm{mt}$ of the macaque. Neuron, 39:681-691.

Morrone, M., Tosetti, M., Montanaro, D., Fiorentini, A., Cioni, G., and Burr, D. (2000). A cortical area that responds specifically to optic flow, revealed by fMRI. Nature Neuroscience, 3(7):1322 - 1328.

Oliva, A. and Torralba, A. (2006). Building the gist of a scene: the role of global image features in recognition. Progress in Brain Res.: Visual Perception, 155:23-26.

Orban, G., Lagae, L., Verri, A., Raiguel, S., Xiao, D., Maes, H., and Torre, V. (1992). First-order analysis of optical flow in monkey brain. PNAS, 89(7):2595-2599.

Rodrigues, J. and du Buf, J. (2006). Multi-scale keypoints in V1 and beyond: object segregation, scale selection, saliency maps and face detection. BioSystems, 2:7590.

Rodrigues, J. and du Buf, J. (2009a). A cortical framework for invariant object categorization and recognition. Cognitive Processing, 10(3):243-261.

Rodrigues, J. and du Buf, J. (2009b). Multi-scale lines and edges in $\mathrm{v} 1$ and beyond: brightness, object categorization and recognition, and consciousness. BioSystems, 95:206-226.

Smith, A., Wall, M., Williams, A., and Singh, K. (2006). Sensitivity to optic flow in human cortical areas $\mathrm{mt}$ and mst. European Journal of Neuroscience, 23(2):561-569.

Wall, M. and Smith, A. (2008). The representation of egomotion in the human brain. Current Biology, 18:191194.

Warren, P. and Rushton, S. (2009). Optic flow processing for the assessment of object movement during ego movement. Current Biology, 19(19):1555-1560.

William, K. and Charles, J. (2008). Cortical neuronal responses to optic flow are shaped by visual strategies for steering. Cerebral Cortex, 18(4):727-739.

Wurtz, R. (1998). Optic flow: A brain region devoted to optic flow analysis? Current Biology, 8(16):R554 R556. 